\title{
A Versatile Dye-Laser Generator-Amplifier System for Intense, Tunable Picosecond Pulse Generation
}

\author{
P. Sperber, M. Weidner, and A. Penzkofer \\ Naturwissenschaftliche Fakultät II - Physik, Universität, \\ D-8400 Regensburg, Fed. Rep. Germany
}

Received 9 October 1986/Accepted 24 October 1986

\begin{abstract}
Passively mode-locked ruby-laser pulses are used to generate nearly diffractionlimited picosecond light pulses in a dye cell by longitudinally amplified spontaneous emission. The output pulses are amplified in three longitudinally pumped dye cells, then spectrally filtered with a grating spectrometer and finally reamplified in a fourth dye amplifier in order to generate intense frequency tunable picosecond light pulses.
\end{abstract}

PACS: $42.55 \mathrm{M}, 42.60$

Various pulsed dye laser systems are available for tunable picosecond pulse generation [1,2]. Flashlamp pumped mode-locked dye lasers [3], synchronously pumped lasers [4], short-cavity resonators [5], quenched transient lasers [6], distributed feedback lasers [7], and amplified spontaneous emission systems [8] have been investigated.

The arrangements applying amplified spontaneous emission (ASE) may be grouped into longitudinally pumped [8-10], transversally pumped [11-13], and travelling-wave transversally pumped systems [14-18]. In longitudinally pumped systems the amplification of spontaneous emission is limited by excitedstate absorption of the pump laser light [19]. In transversally excited dye cells the duration of the amplified spontaneous emission signal is restricted by the transit time of light through the pumped region, and two pulses are emitted in opposite directions. The travelling-wave transverse pumping technique uses a grating for matching the propagation of the pump pulse and the amplified spontaneous emission signal. It avoids the drawbacks of longitudinal (excited-state absorption of pump pulse) and transverse pumping (limitation of duration, double pulsing).

In this paper we describe a versatile longitudinally pumped dye laser generator-amplifier system that generates intense frequency tunable picosecond light pulses and avoids the problems of pump-pulse excitedstate absorption. The light generation is initiated by amplified spontaneous emission in a dye generator cell. The output signal is amplified in a chain of longitudinal amplifiers, and spectrally narrowed and tuned with a spectrometer. Overall conversion efficiencies (output dye-laser pulse energy to total input pump pulse energy) of about $10 \%$ are achieved.

\section{Amplification Considerations}

The light generation in a longitudinally pumped dye generator cell is limited by excited-state absorption. Pump-laser photons that are absorbed by excited-state absorption do not contribute to amplification of spontaneous emission. Even under extreme bleaching conditions (pump energy density $w_{L} \gg \varepsilon_{S, L}=h v_{L} / \sigma_{L}$; $\varepsilon_{S, L}$ is saturation energy of dye bleaching [dimension $\left.\mathrm{J} / \mathrm{cm}^{2}\right], \sigma_{L}$ is ground-state absorption cross-section at the pump-laser frequency $v_{L}$ ) the pump pulse penetration depth is limited to

$l_{\text {eff }}=\left(N \sigma_{\text {ex }, L}\right)^{-1}$.

This equation is derived from

$T_{\text {ex }, L}=\exp \left(-\int_{0}^{l} N_{\text {ex }} \sigma_{\text {ex }, L} d z\right)=\exp \left(-N \sigma_{\text {ex }, L} l_{\text {eff }}\right)=\mathrm{e}^{-1}$ 
and is valid for sample lengths $l>l_{\text {eff. }} N_{\text {ex }}$ is the population number density of the $S_{1}$ state of the dye. $N$ is the total number density of dye molecules, $\sigma_{\mathrm{ex}, L}$ is the excited-state absorption cross-section at the pump laser frequency.

The amplification of spontaneous emission is given by the differential equation [19]

$\frac{\partial I_{F}}{\partial z}=\frac{h v_{F} N_{\mathrm{ex}} \Delta \Omega_{\mathrm{ASE}}}{\tau_{\mathrm{rad}} 4 \pi}+\left(\sigma_{\mathrm{em}}-\sigma_{\mathrm{ex}, F}\right) N_{\mathrm{ex}} I_{F}$.

The first term gives the spontaneous emission and the second term represents the amplification of spontaneous emission. $\tau_{\text {rad }}$ is the radiative lifetime of the excited-state. It is related to the fluorescence lifetime $\tau_{F}$ by $\tau_{F}=q_{F} \tau_{\mathrm{rad}}$, where $q_{F}$ is the fluorescence quantum efficiency. Spontaneous emission radiates in all directions (solid angle $\Delta \Omega=4 \pi$ ) but amplification of spontaneous emission is important only within a small solid angle $\Delta \Omega_{\mathrm{ASE}}=\left(\pi d_{F}^{2} / 4\right) / L^{2}$ where $d_{F}$ is the beam diameter of the amplified spontaneous emission signal at a distance $L$ from the generator cell.

Integration of (2) gives

$$
\begin{aligned}
I_{F, \mathrm{ASE}}= & \frac{h v_{F} \Delta \Omega_{\mathrm{ASE}}}{4 \pi \tau_{\mathrm{rad}}\left(\sigma_{\mathrm{em}}-\sigma_{\mathrm{ex}, F}\right)} \\
& \times\left\{\exp \left[\left(\sigma_{\mathrm{em}}-\sigma_{\mathrm{ex}, F}\right) \int_{0}^{l} N_{\mathrm{ex}} d z\right]-1\right\} .
\end{aligned}
$$

The maximum possible intensity is obtained for $\int_{0}^{l} N_{\mathrm{ex}} d z=N l_{\mathrm{eff}}=\sigma_{\mathrm{ex}, L}^{-1}$

$$
\begin{aligned}
I_{F, \mathrm{ASE}}^{\max }= & \frac{h v_{F} \Delta \Omega_{\mathrm{ASE}}}{4 \pi \tau_{\mathrm{rad}}\left(\sigma_{\mathrm{em}}-\sigma_{\mathrm{ex}, F}\right)} \\
& \times\left\{\exp \left[\left(\sigma_{\mathrm{em}}-\sigma_{\mathrm{ex}, F}\right) / \sigma_{\mathrm{ex}, L}\right]-1\right\} .
\end{aligned}
$$

The spontaneous intensity is, see (2) without second term, $I_{\mathrm{sp}}=h v_{F} \Delta \Omega_{\mathrm{ASE}} \int_{0}^{l} N_{\mathrm{ex}} d z /\left(4 \pi \tau_{\mathrm{rad}}\right)$. The maximum spontaneous intensity is limited to $I_{\mathrm{sp}}^{\mathrm{max}}=h v_{F} \Delta \Omega_{\mathrm{ASE}} /$ $\left(4 \pi \sigma_{\mathrm{ex}, L} \tau_{\mathrm{rad}}\right)$.

The maximum amplification factor $G_{\mathrm{ASE}}^{\max }=I_{F, \mathrm{ASE}}^{\max } / I_{\mathrm{sp}}^{\max }$ is

$G_{\mathrm{ASE}}^{\max }=\frac{\exp \left[\left(\sigma_{\mathrm{em}}-\sigma_{\mathrm{ex}, F}\right) / \sigma_{\mathrm{ex}, L}\right]-1}{\left(\sigma_{\mathrm{em}}-\sigma_{\mathrm{ex}, F}\right) / \sigma_{\mathrm{ex}, L}}$.

This maximum gain is already obtained for a picosecond pump pulse energy density of about

$w_{L, \mathrm{opt}}=h v_{L} N l_{\mathrm{eff}}=\frac{h v_{L}}{\sigma_{\mathrm{ex}, L}}$.

Since further increase of pump energy does not increase the amplification of fluorescence light, only the optimum pump pulse energy is entered to the generator cell in the experiments. The residual pump pulse energy is used to pump longitudinally a series of amplifier cells to increase the output signal of the generator cell to a high energy level.

The small-signal amplification of input light in amplifier cells is also determined by (2) where the first term may be neglected. The output signal is given by

$I_{F, \text { out }}=I_{F, \text { in }} \exp \left[\left(\sigma_{\mathrm{em}}-\sigma_{\mathrm{ex}, F}\right) \int_{0}^{l} N_{\mathrm{ex}} d z\right]$.

The maximum possible small-signal gain is $\left(\int_{0}^{l} N_{\mathrm{ex}} d z\right.$

$\left.=N l_{\mathrm{eff}}=\sigma_{\mathrm{ex}, L}^{-1}\right)$

$G_{\mathrm{AMP}}^{\max }=\frac{I_{F, \text { out }}^{\max }}{I^{F, \text { in }}}=\exp \left[\left(\sigma_{\mathrm{em}}-\sigma_{\mathrm{ex}, F}\right) / \sigma_{\mathrm{ex}, L}\right]$.

The minimal necessary pump pulse energy for maximum small-signal amplification is again given by $W_{L, \text { opt }}$ of (6).

The ratio of amplifier gain to generator gain is

$$
\begin{aligned}
\frac{G_{\mathrm{AMP}}}{G_{\mathrm{ASE}}} & =\frac{\left(\sigma_{\mathrm{em}}-\sigma_{\mathrm{ex}, F}\right) \int_{0}^{l} N_{\mathrm{ex}} d z}{1-\exp \left[-\left(\sigma_{\mathrm{em}}-\sigma_{\mathrm{ex}, F}\right) \int_{0}^{l} N_{\mathrm{ex}} d z\right]} \\
& =\frac{\ln \left(G_{\mathrm{AMP}}\right)}{1-G_{\mathrm{AMP}}^{-1}} .
\end{aligned}
$$

For maximum amplification the ratio is

$$
\begin{aligned}
\frac{G_{\mathrm{AMP}}^{\max }}{G_{\mathrm{ASE}}^{\max }} & =\frac{\left(\sigma_{\mathrm{em}}-\sigma_{\mathrm{ex}, F}\right) / \sigma_{\mathrm{ex}, L}}{1-\exp \left[-\left(\sigma_{\mathrm{em}}-\sigma_{\mathrm{ex}, F}\right) / \sigma_{\mathrm{ex}, L}\right]} \\
& =\frac{\ln \left(G_{\mathrm{AMP}}^{\max }\right)}{1-\left(G_{\mathrm{AMP}}^{\max }\right)^{-1}} .
\end{aligned}
$$

The amplification of input light dominates over the intrinsic amplified spontaneous emission as long as

$I_{F, \text { in }}>I_{\mathrm{sp}} \frac{1-G_{\mathrm{AMP}}^{-1}}{\ln \left(G_{\mathrm{AMP}}\right)}$.

A seeding signal of intensity $I_{F, \text { in }}$ determined by (11) is enough to dominate over amplification of spontaneous emission. In our experiments the seeding signal from the generator cell dominates already completely in the first amplifier cell.

As along the amplifier chain the light signal is amplified the small-signal amplification changes over to the strong-signal amplification where the gain is limited by depopulation of the excited state by stimulated emission. The strong signal amplification has to be $G_{\text {strong }}<\left(v_{F} / v_{L}\right) W_{L} / W_{F \text {, in }}$ by reason of energy conservation. In the strong-signal amplification case the usable pump pulse energy is not limited to $W_{L, \text { opt }}$ since it is used to reexcite molecules returned to the groundstate by stimulated emission. 


\section{Experimental Arrangement}

The experimental set-up of the ruby-laser pumped dyelaser generator-amplifier system is depicted in Fig. 1. A passively mode-locked ruby laser (saturable dye DDI in methanol) generates a train of picosecond pulses. A krytron triggered Kerr shutter [20] selects a single pulse out of the train. The separated pulse is increased in energy by passing twice through a ruby amplifier. The amplified ruby-laser pulses have an average duration of $30 \mathrm{ps}$ and a pulse energy of up to $6 \mathrm{~mJ}$.

The amplified ruby-laser pulse is slightly focused (lens $\mathrm{L} 1$ focal length $f=1 \mathrm{~m}$ ) and pumps the dye-laser generator-amplifier system. The mirror M1 (reflectivity $R=30 \%$ ) selects the pump pulse for generating amplified spontaneous emission in the generator cell G. The generated signal is amplified in three following longitudinally pumped dye cells. The laser mirrors M2 $(R=30 \%), \mathrm{M} 3(R=50 \%)$ and a $90^{\circ}$ deflection prism direct the pump light to the amplifier cells. The edge filters E reflect ruby light while transmitting the amplified spontaneous emission signal. The temporal synchronization of the ruby-laser pump pulses to the dye-laser signal is achieved by observing the temporal output with a streak camera. Without temporal overlap between pump pulse and signal from the generator cell the amplifiers act themselves as generators and produce amplified spontaneous emission.
The optimum time synchronization is adjusted by pulse duration minimization.

In the generator cell $\mathrm{G}$ and the first amplifier cell A1 stimulated resonance Raman scattering [21, 22] may occur simultaneously producing a spectrally small line upon the broad amplified spontaneous signal. The amplified spontaneous emission signal may act as seeding signal for stimulated resonance Raman amplification (start of stimulated Raman scattering from amplified spontaneous emission signal instead of quantum fluctuations). The amplification of the stimulated resonance Raman signal in the amplifier chain would reduce the amplification of the broad-band amplified fluorescence signal. Special edge filters EF are used to suppress the resonance Raman signal at the cost of some narrowing of the spectral width of the ampified spontaneous emission signal.

The spectral tuning and narrowing of the generated dye-laser signal is achieved with a grating spectrometer arrangement (S1, L3, GR, L4, S2). The energy loss due to spectral narrowing is compensated in a final dye cell amplifier A4 which is pumped with a split-off (BS before L1) and amplified (amplifier 2) part of the rubylaser pulse. The increase of the ruby-laser pulse energy in amplifier 2 is about of factor of 10 (single pass).

The generated dye-laser pulses are analysed with a streak camera (SC) and a spectrograph (SP). Various photodetectors (not shown in Fig. 1) along the

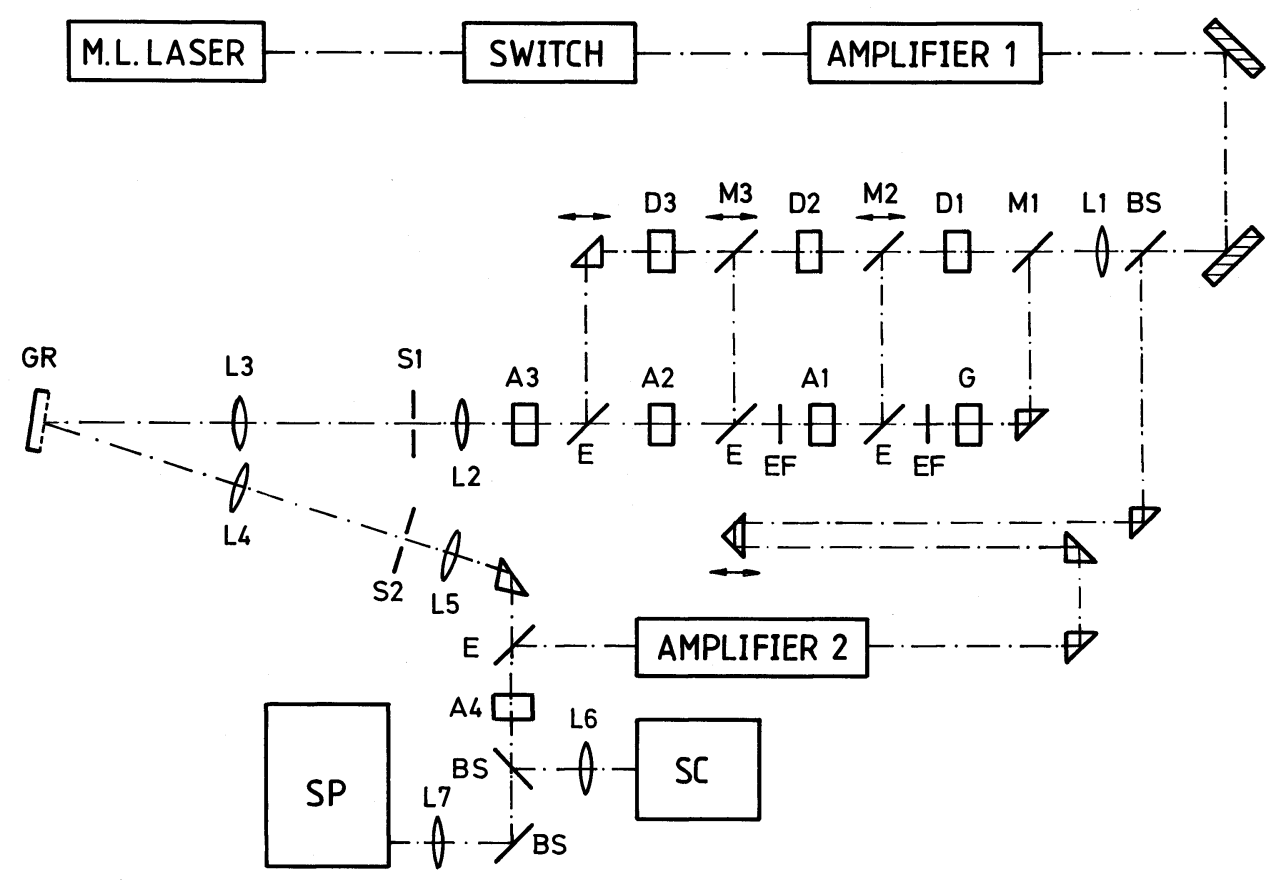

Fig. 1. Experimental set-up of ruby-laser pumped dye-laser generator-amplifier system. (BS: Beam splitter. L1-L7: lenses. M1-M3: partial reflecting mirrors. G: generator dye cell. A1-A4: amplifier dye cells. D1-D3: optical delay blocks. E: longpass edge filters. EF: short-pass edge filters. GR: grating. SP: $60 \mathrm{~cm}$ grating spectrograph. SC: streak camera) 
generator-amplifier chain are used to monitor the amplification behaviour. The divergence of the dyelaser pulses behind the generator and the various amplifiers is analysed with a diode array system and with apertured photodetectors.

\section{Results}

In our experiments various laser dyes have been used to cover the spectral range from 725 to $940 \mathrm{~nm}$ (rhodamine 800 in methanol: $725-790 \mathrm{~nm}$; DDI in methanol: $745-755 \mathrm{~nm}$; styril 9 in DMSO: 790-880 nm; HDITC in 1:1 ethylene glycol-DMSO mixture: $825-870 \mathrm{~nm}$; IR140 in 1:1 ethylene glycolDMSO mixture: $880-940 \mathrm{~nm}$ ). The dye IR140 is from Kodak, the other dyes are from Lambda Physik. Here only results for the dye DDI $\left(1,1^{\prime}\right.$-diethyl$2,2^{\prime}$ dicarbocyanine iodide) in methanol are presented.

The absorption and emission spectrum of DDI in methanol is depicted in Fig. 2. The absorption crosssections are obtained from transmission measurements. The stimulated emission cross sections are derived from backward fluorescence measurements [23-27] (excitation source: tungsten lamp; detection with Tracor DARRS diode array system). Other dye parameters are listed in Table 1. In the experiments the used dye concentrations are $10^{-4} \mathrm{~mol} / 1$ for the generator cell (length $1 \mathrm{~cm}$ ) and $2 \times 10^{-4} \mathrm{~mol} / 1$ for the amplifier cells (lengths $0.5 \mathrm{~cm}$ ).

The ratio of generated output pulse energy to total pump pulse energy in front of the beam splitter BS is plotted in Fig. 3 for the generator cell $G$ and the amplifiers A1, A2, and A3. The detectors were aperture to accept a divergence angle of $\Delta \theta=2 \times 10^{-3} \mathrm{rad}$ (solid
Table 1. Parameters of DDI in methanol. Concentration $C=10^{-4} \mathrm{~mol} / 1$, laser wavelength $\lambda_{L}=694.3 \mathrm{~nm}$

\begin{tabular}{|c|c|c|}
\hline Parameter & Value & Ref. \\
\hline \multicolumn{3}{|c|}{ Cross-sections } \\
\hline$\sigma_{L}$ & $7.5 \times 10^{-16} \mathrm{~cm}^{2}$ & a \\
\hline$\sigma_{\mathrm{ex}, L}$ & $5 \times 10^{-17} \mathrm{~cm}^{2}$ & {$[28]$} \\
\hline$\sigma_{\mathrm{ex}, \boldsymbol{F}}$ & $5 \times 10^{-17} \mathrm{~cm}^{2}$ & \\
\hline \multicolumn{3}{|c|}{$\begin{array}{l}\text { Maximum penetration } \\
\text { depth }\end{array}$} \\
\hline$l_{\text {eff }}$ & $0.33 \mathrm{~cm}$ & Eq. (1) \\
\hline \multicolumn{3}{|l|}{ Lifetimes } \\
\hline$\tau_{F}$ & $17 \mathrm{ps}$ & {$[28]$} \\
\hline$\tau_{\mathrm{rad}}$ & $4.0 \mathrm{~ns}$ & \\
\hline \multicolumn{3}{|c|}{ Resonance Raman shift } \\
\hline$\tilde{v}_{R}$ & $1348 \mathrm{~cm}^{-1}$ & d \\
\hline
\end{tabular}

${ }^{a}$ Fig. 2

b Assumed

c Calculated with Strickler-Berg formula [24]

d This work

angle $\Delta \Omega=\pi \Delta \theta^{2} / 4=3 \times 10^{-6} \mathrm{sr}$ ) which is slightly larger than the divergence of the amplified spontaneousemission signal behind the generator cell and of the amplified signal behind the third amplifier. The pump radiation enters convergent to the generator cell with a beam diameter of $d_{G} \approx 0.8 \mathrm{~mm}$ (FWHM). For the amplifier cells the pump pulse is slightly divergent with diameters $d_{\mathrm{A} 1} \simeq 0.4 \mathrm{~mm}, d_{\mathrm{A} 2} \simeq 0.6 \mathrm{~mm}$, and $d_{\mathrm{A} 3} \simeq 0.8 \mathrm{~mm}$. The generated radiation is polarized parallel to the pump pulse polarization.

The curves in Fig. 3 represent a least-square fit to the experimental points. The open circles show the build-up of amplified spontaneous emission in the generator cell. For a weak total pump pulse energy



Fig. 2. Absorption cross-section $\sigma_{\mathrm{abs}}$ and stimulated emission cross-section $\sigma_{\mathrm{em}}$ spectra of dye DDI in methanol. (Molar decadic coefficients are obtained by $\varepsilon=\sigma N_{A} /\left[1000 \mathrm{~cm}^{3} \ln (10)\right], N_{A}$ is Avogado's constant). Structure formula of DDI is inserted 


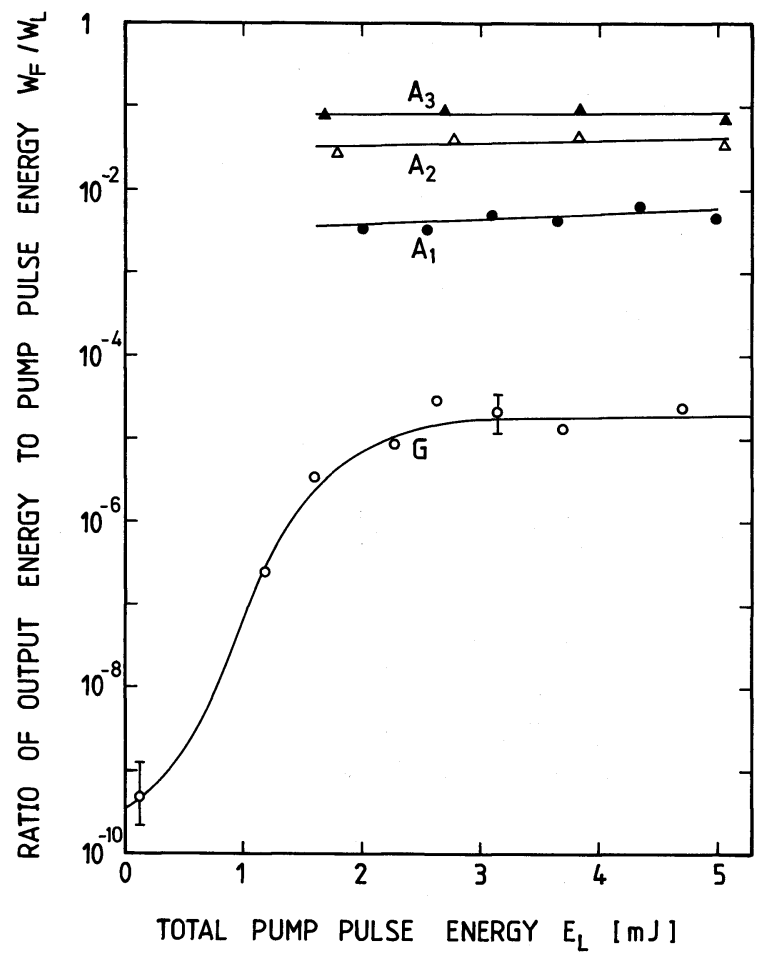

Fig. 3. Energy conversion of ruby pump light $W_{L}$ to dye laser light $W_{F}$. The total pump pulse energy is measured before entering the generator-amplifier chain (position L1 in Fig. 1). The curves connect the experimental points smoothly. Open circles, signal behind generator G. Closed circles, signal behind amplifier A1. Open triangles, pulse behind second amplifier A2. Closed triangles, output behind third amplifier A3

$\left(W_{L}<100 \mu \mathrm{J}\right)$ only spontaneous emission occurs. The experimental point at $100 \mu \mathrm{J}$ with $W_{F} / W_{L} \approx 5 \times 10^{-10}$ is in good agreement with expectation of spontaneous radiation $\left[W_{\mathrm{sp}} / W_{L}=\left(v_{F} / v_{L}\right) \quad\left(\tau_{F} / \tau_{\mathrm{rad}}\right)\right.$ $R_{1} \Delta \Omega / 4 \pi \approx 3 \times 10^{-10} ; \quad \lambda_{F} \approx 740 \mathrm{~nm}, \quad \lambda_{L}=694.3 \mathrm{~nm}$, $R_{1}=0.3$ reflectivity of mirror $\left.\mathrm{M} 1, \Delta \Omega=3 \times 10^{-6} \mathrm{sr}\right]$. With increasing pump energy the amplified spontaneous emission signal rises steeply up to a limiting value of $W_{F} / W_{L} \simeq 2 \times 10^{-5}$ giving an amplification of the fluorescence of $G_{\mathrm{ASE}} \approx 4 \times 10^{4}$. The expected maximum amplification according to (5) is $G_{\mathrm{ASE}}^{\max } \approx 8 \times 10^{4}$ for $\sigma_{\mathrm{em}}=7.5 \times 10^{-16} \mathrm{~cm}^{2} \quad$ (Fig. 2), $\sigma_{\text {ex }, F}=5 \times 10^{-17} \mathrm{~cm}^{2}$, and $\sigma_{\text {ex }, L}=5 \times 10^{-17} \mathrm{~cm}^{2}[28]$.

The dye amplifier chain increases the output pulse signal up to an energy conversion of $W_{F} / W_{L} \simeq 0.09$ after the third amplifier cell (Fig. 3). In the saturation region $\left(W_{L}>2 \mathrm{~mJ}\right)$ the amplification in cell $\mathrm{A} 1$ is $\approx 200$, in cell $\mathrm{A} 2 \approx 8$, and in cell $\mathrm{A} 3 \approx 2$. The pulse to pulse energy fluctuations behind the amplifier chain are very small.

The spatial distribution of the amplified spontaneous emission signal behind the generator cell is depicted in Fig. 4. It exhibits a sharp spike of divergence $\Delta \theta=2 \theta_{1 / 2}=(1 \pm 0.4) \times 10^{-3} \mathrm{rad}$ (corresponding solid angle $\Delta \Omega_{\mathrm{ASE}}=\pi \Delta \Theta^{2} / 4 \simeq 8 \times 10^{-7}$ sr) upon a broad

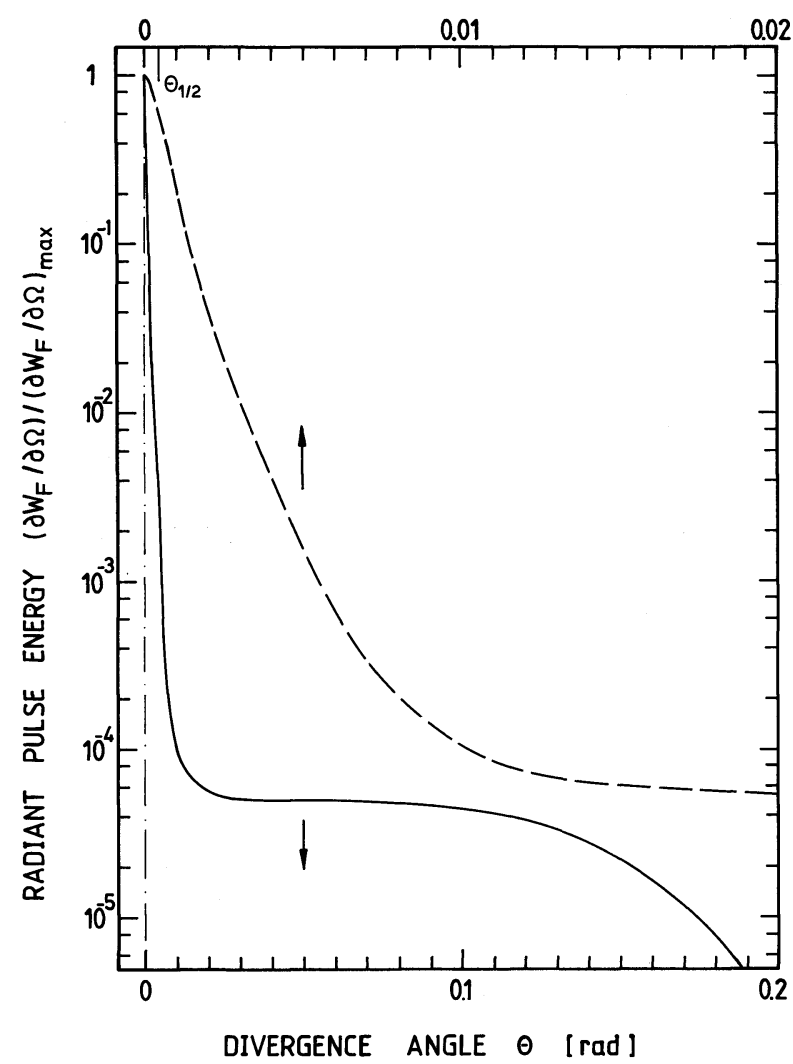

Fig. 4. Angular distribution of output signal of laser generator cell in the saturation region $\left(W_{L} \simeq 3 \mathrm{~mJ}\right)$. The upper curve is a factor of 10 expanded

pedestal (see also [29]). The central spike is a factor of $2 \times 10^{4}$ more intense than the pedestal and contains about one fifth of the total amplified spontaneous emission energy. The pedestal radiant pulse energy is about a factor of 200 higher than the sideward fluorescence radiance $\partial W_{\mathrm{sp}} / \partial \Omega$. The divergence angle $\Delta \theta$ of the sharp amplified spontaneous emission spike is considerably less than the geometrical angle $d_{G} / l_{\text {eff }} \simeq 0.25$. In the experiments the pump pulses traverses convergently the generator cell. Divergent pump pulse geometries give larger divergence of the amplified spontaneous emission signal [8-10]. The small divergence is thought to be due to the build-up of spatial coherence (interference of amplifying photons with fluorescing molecules) in the process of amplified spontaneous emission $[30,31]$. It should be noted that in the saturation region of amplified spontaneous emission $\left(W_{\mathrm{L}} \gtrsim 2 \mathrm{~mJ}, W_{F} \gtrsim 4 \times 10^{-8} \mathrm{~J}, I_{F}=W_{F} /\left(d_{G}^{2} \Delta t_{F}\right)\right.$ $\left.\simeq 7 \times 10^{6} \mathrm{~W} / \mathrm{cm}^{2}\right)$ the number of photons which transverse the area of coherence $S$ at $l_{\text {eff }}\left[S \simeq l_{\text {eff }}^{2}\left(d_{G}^{2} \tilde{v}_{F}^{2}\right)\right.$ $\left.\approx 10^{-7} \mathrm{~cm}^{2}\right]$ within the coherence time $t_{\text {coh }}\left[t_{\text {coh }} \simeq 1 /\right.$ $\left.\Delta v_{F}=1 /\left(c \Delta \tilde{v}_{F}\right) \simeq 2.8 \times 10^{-13} \mathrm{~s}, \quad \tilde{v}_{F} \simeq 120 \mathrm{~cm}^{-1}\right] \quad$ is $\delta=I_{F} S t_{\text {coh }} / h v_{F} \gtrsim 10^{5}$ ( $\delta$ is the photon degeneracy parameter) [32]. 


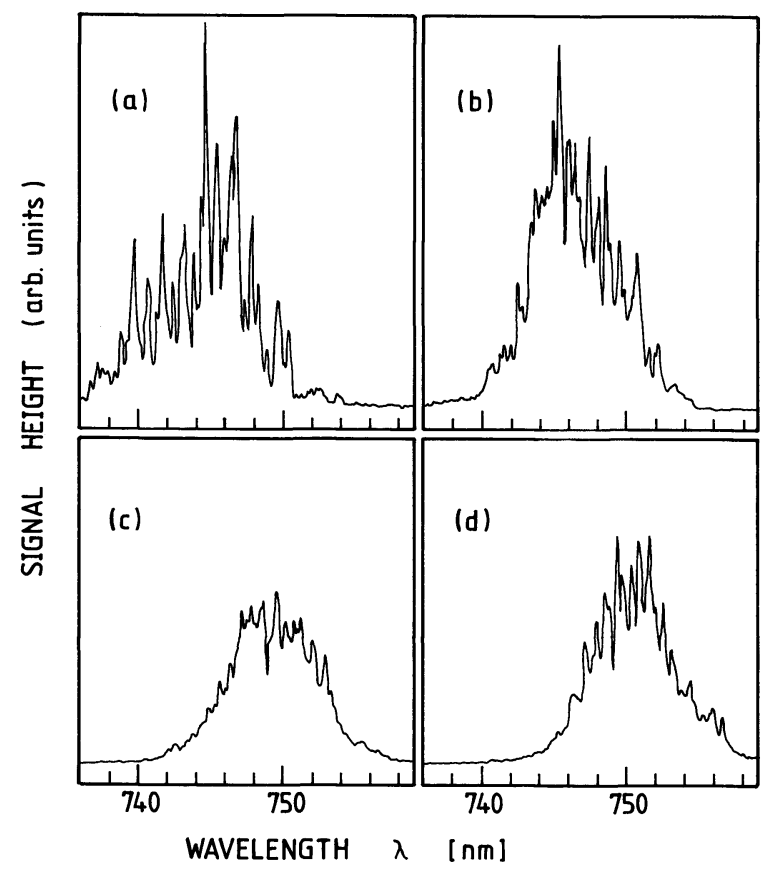

Fig. 5a-d. Generated dye laser spectra in generator-amplifier system. (a) behind generator cell G, (b) behind first amplifier A1, (c) behind second amplifier A2, (d) behind third amplifier A3

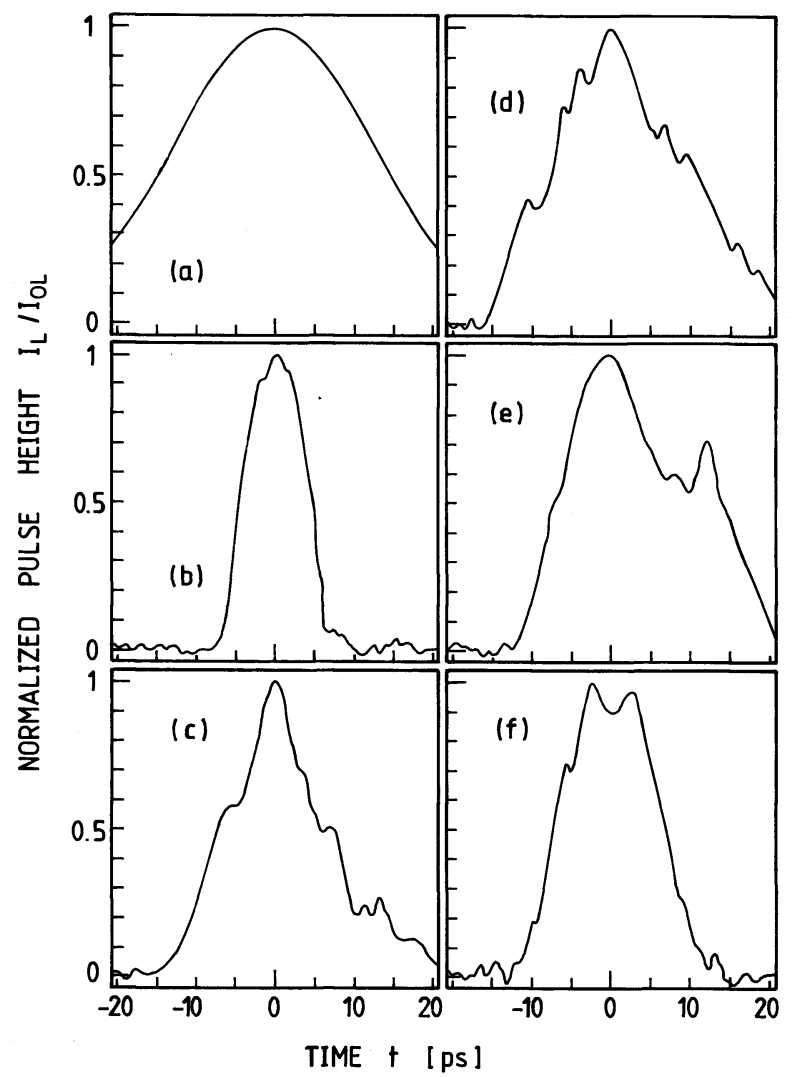

Fig. 6a-f. Temporal pulse shapes of (a) pump pulse, (b) amplified spontaneous emission signal behind generator $G$, (c) behind amplifier A1, (d) behind amplifier A2, (e) behind amplifier A3, and (f) behind amplifier A4
Typical pulse spectra along the generator-amplifier chain are shown in Fig. 5. The generator spectrum (a) is strongly structured. The positions of the spectral peaks change from shot to shot. The structure is thought to be caused by the build-up of temporal coherence in the amplification of spontaneous emission $[30,31]$. The spectral fine structure agrees approximately with the inverse overall duration of the generated signal $\left(\Delta v \approx 1 / \Delta t_{F}\right.$, see Fig. $\left.6 \mathrm{~b}\right)$. Along the amplifiers the spectra are smoothed by gain saturation (Fig. 6b-d). The central emission frequency shifts slightly to longer wavelengths. This behaviour is mainly due to the transmission characteristics of the long-pass edge filters E. The spectral overall width of the generated pulses is approximately $\Delta \tilde{v}_{F} \simeq 120 \mathrm{~cm}^{-1}$ (FWHM).

The temporal pulse development is illustrated in Fig. 6. The pulse durations are measured with a Hamamatsu type C1587 streak camera with fast streak plug-in of type M1952 (2 ps time resolution). Part (a) depicts the input pump pulse shape $\left(\Delta t_{L}=30 \mathrm{ps}\right.$ FWHM). The temporal pulse shape of the amplified fluorescence signal behind the generator cell is shown in part (b) (pump pulse energy $W_{L} \simeq 3 \mathrm{~mJ}$ ). The overall duration is $\Delta t_{F} \simeq 9 \mathrm{ps}$ (FWHM). The generation of pulse durations shorter than the pump pulse duration is due to the high amplification of fluorescence light [14-19] and is supported by the short fluorescence lifetime of the dye. The amplifiers A1 to A3 broaden the pulse duration from $9 \mathrm{ps}$ after the generator to about 20 ps after the third amplifier (Fig. 6c-e). The broadening is due to gain saturation in the amplifiers. A temporal substructure of the pulses is resolved. An even finer temporal structure is expected from the spectral distributions of Fig. 5 (temporal beating structure).

The generated picosecond dye laser pulses in the generator-amplifier system $\mathrm{G}-\mathrm{A} 3$ are not bandwidth limited $(\Delta v \Delta t \simeq 70)$ because of the broad region of stimulated emission. With a grating spectrometer arrangement (Fig. 1) the pulses may be spectrally narrowed down to $\Delta \tilde{v} \simeq 2 \mathrm{~cm}^{-1}(\Delta v \Delta t \simeq 1)$ without remarkable temporal broadening (for band-width limited Gaussian pulses it is $\Delta v \Delta t=0.441$ ). In our experiments we narrowed the spectra down to $\Delta \tilde{v}_{F} \simeq 25 \mathrm{~cm}^{-1}$ (focal length of lenses L3 and L4 is $30 \mathrm{~cm}$, grating has 1200 lines $/ \mathrm{mm}$, slit widths of $S_{1}$ and $\mathrm{S}_{2}$ are $\left.1 \mathrm{~mm}\right)$ and $\Delta \tilde{v}_{F} \simeq 8 \mathrm{~cm}^{-1}(0.2 \mathrm{~mm}$ slit widths $)$ and tuned the frequency within the input band width. The energy losses due to spectral narrowing, due to tuning to the spectral wings, and due to transmission losses are compensated in the final amplifier cell A4.

The results obtained with the spectrometer and reamplifier arrangement are summarized in Fig. 7 where the normalized spectral output pulse energy density envelopes $\left(\partial W_{F} / \partial \lambda_{F}\right) / W_{L}$ are plotted versus 


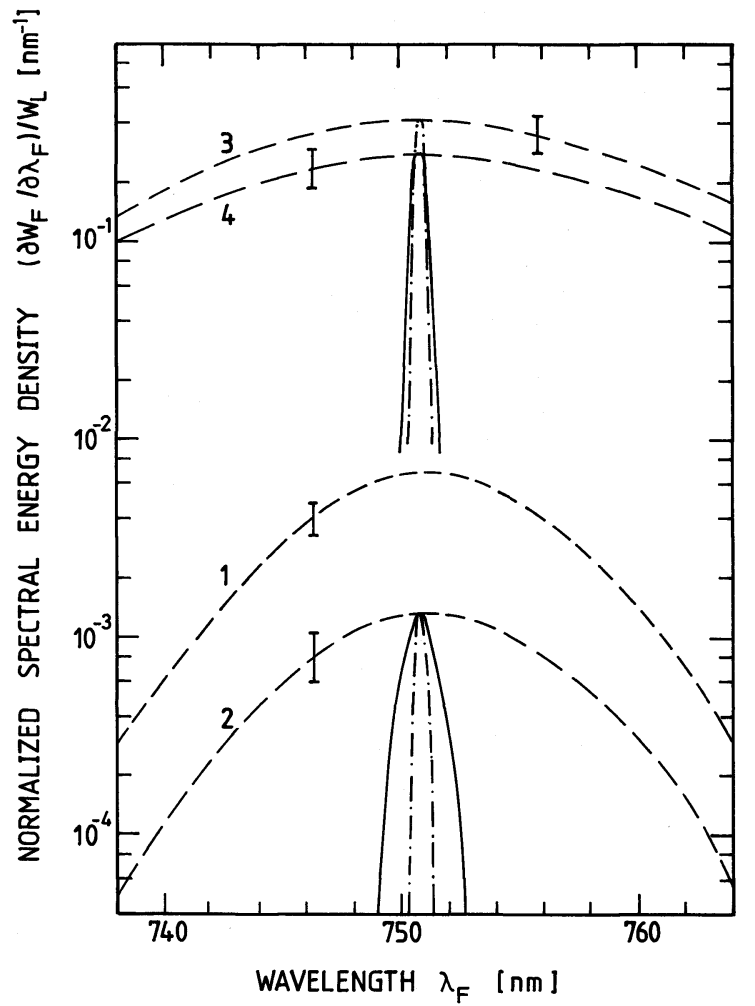

Fig. 7. Spectral distribution of generated light pulses. (Dashed curves: 1 spectrum behind amplifier A3 before spectral narrowing; 2 envelope of the peaks of narrowed pulses behind spectrometer arrangement; 3 and 4 envelopes of the peaks of strongly narrowed and weakly narrowed pulses behind amplifier A4, respectively). Solid curves show weakly narrowed pulse spectra. Dash-dotted curves present strongly narrowed pulse spectra

wavelength for the pulses entering the spectrometer (curve 1), leaving the spectrometer (curve 2) and leaving the amplifier (curve 4 for weakly narrowed pulses and curve 3 for strongly narrowed pulses). The light passage through the spectrometer system cause a transmission loss of 0.2 (comparison of curves 1 and 2). At the central frequency the spectral pulse shapes of weakly and strongly narrowed pulses behind the spectrometer and behind dye amplifier A4 are included. The amplifier slightly narrows the spectral pulse width because the spectral components are spatially dispersed and the amplifier has a spatial bell shaped gain profile. In the central frequency region the pulse amplification is about a factor of 150 and a conversion efficiency of $W_{F} / W_{L} \approx 0.15$ and 0.05 is obtained for the weakly and strongly narrowed pulses, respectively ( $W_{L}$ ruby pulse energy at lens L1, Fig. 1). At the spectral wings the amplification rises up to a factor of 3000 .

A temporal pulse structure of a weakly narrowed and amplified pulse is shown in Fig. 6f. The pulse seems still to be somewhat structured. Its envelope duration is $\Delta t_{F}=15 \mathrm{ps}$. For the strongly narrowed and amplified pulses durations of $\Delta t_{L} \approx 20$ ps are measured.

\section{Conclusions}

The reported dye-laser generator-amplifier system longitudinally pumped with single picosecond laser pulses allows the generation of frequency tunable, nearly bandwidth and diffraction limited, intense picosecond light pulses. Using a mode-locked ruby pump laser and several laser dyes the spectral range from 725 to $940 \mathrm{~nm}$ was covered. The spectral range may be extended further to the infrared by applying suitable dyes $[33,34]$. Using second-harmonic pulses of the picosecond ruby laser and suitable dyes, frequency tunable picosecond pulses in the region of 380-700 nm should be producable with the described arrangement. Instead of the mode-locked ruby laser, mode-locked $\mathrm{Nd}$-glass or Nd-Yag lasers and their harmonics may be used as pump sources for the described dye-laser generator-amplifier system.

\section{References}

1. S.L. Shapiro (ed.): Ultrashort Light Pulses, Topics Appl. Phys. 18 (Springer, Berlin, Heidelberg 1977)

2. F.P. Schäfer (ed.): Dye Lasers, 2nd ed., Topics Appl. Phys. 1 (Springer, Berlin, Heidelberg 1977)

3. D.J. Bradley, F. O'Neill: Opto-Electronics 169 (1969)

4. L.S. Goldberg, C.A. Moore: Appl. Phys. Lett. 27, 217 (1975)

5. A.J. Cox, G.W. Scott, L.P. Talley: Appl. Phys. Lett. 31, 389 (1977)

6. S. Szatmári, F.P. Schäfer: Appl. Phys. B33, 95 (1984)

7. Zs. Bor, A. Müller, B. Rácz, F.P. Schäfer: Appl. Phys. B27, 77 (1982)

8. M.E. Mack: Appl. Phys. Lett. 15, 166 (1969)

9. A.N. Rubinov, M.C. Richardson, K. Sala, A.J. Alcock: Appl. Phys. Lett. 27, 358 (1975)

10. W. Falkenstein, A. Penzkofer, W. Kaiser: Opt. Commun. 27, 151 (1978)

11. S.L. Chin, G. Bedard: Opt. Commun. 4, 148 (1971)

12. C. Lin, T.K. Gustafson, A. Dienes: Opt. Commun. 8, 210 (1973)

13. A. Penzkofer: Appl. Phys. B40, 85 (1986)

14. H.J. Polland, T. Elsaesser, A. Seilmeier, W. Kaiser: Appl. Phys. B32, 53 (1983)

15. Zs. Bor, S. Szatmári, A. Müller: Appl. Phys. B32, 101 (1983)

16. S. Szatmári, F.P. Schäfer: Opt. Commun. 49, 281 (1984)

17. T. Elsaesser, H.J. Polland, A. Seilmeier, W. Kaiser: IEEE J. QE-20, 191 (1984)

18. W. Lee, C. Ning, Z. Huang: Appl. Phys. B40, 35 (1986)

19. A. Penzkofer, W. Falkenstein: Opt. Quantum Electron. 10, 399 (1978)

20. A. Penzkofer, S. Weinmann, J. Biebl: J. Phys. E 14, 1411 (1981)

21. Ya.S. Bobovich, A.V. Bortkevich: Opt. Spectrosc. 26, 578 (1969)

22. H.J. Weigmann, M. Pfeiffer, A. Lau, K. Lenz: Opt. Commun. 12, 231 (1974) 
23. O.G. Peterson, J.P. Webb, W.C. McColgin, J.H. Eberly: J. Appl. Phys. 42, 1917 (1971)

24. S.J. Strickler, R.A. Berg,: J. Chem. Phys. 37, 814 (1962)

25. J.B. Birks, D.J. Dyson: Proc. Roy. Soc. London A 275, 135 (1963)

26. A. Penzkofer, P. Sperber: Chem. Phys. 88, 309 (1984)

27. A. Penzkofer, Y. Lu: Chem. Phys. 103, 399 (1986)

28. W. Blau, R. Reber, A. Penzkofer: Opt. Commun. 43, 210 (1982)
29. L.D. Derkocheva, V.A. Petukhov: Opt. Spectrosc. 45, 754 (1978)

30. I.L. Klyukach, R.I. Sokolovsku: Sov. Phys. JETP 44, 223 (1977)

31. P. Sperber, A. Penzkofer: To be published

32. L. Mandel, L. Wolf: Rev. Mod. Phys. 37, 231 (1965)

33. Y. Miyazoe, M. Maeda: Opto-Electron. 2, 227 (1979)

34. A. Seilmeier, W. Kaiser, B. Sens, K.H. Drexhage: Opt. Lett. 8, 205 (1983)

Responsible for Advertisements: E. Lückermann, G. Probst, Heidelberger Platz 3, D-1000 Berlin 33, Tel. (0 30) 82 07-1, Telex 01-85411 Responsible for the Text: H. K. V. Lotsch, Tiergartenstrasse 17, D-6900 Heidelberg. Printers: Brühlsche Universitätsdruckerei, Giessen, Printed in Germany (C) by Springer-Verlag Berlin Heidelberg 1987.

Springer-Verlag GmbH \& Co. KG, D-1000 Berlin 33 